Mots. Les langages du politique

Discours de violence au nom de la foi

\title{
Les Ordonnances royales sur les juifs en Espagne et en France
}

Juana Ugarte

\section{(2) OpenEdition \\ Journals}

Édition électronique

URL : https://journals.openedition.org/mots/1328

DOI : $10.4000 /$ mots. 1328

ISSN : 1960-6001

Éditeur

ENS Éditions

Édition imprimée

Date de publication : 1 novembre 2005

Pagination : 35-46

ISBN : 2-84788-084-4

ISSN : 0243-6450

Référence électronique

Juana Ugarte, "Les Ordonnances royales sur les juifs en Espagne et en France », Mots. Les langages du politique [En ligne], 79 | 2005, mis en ligne le 28 mai 2008, consulté le 22 avril 2022. URL : http:// journals.openedition.org/mots/1328; DOI : https://doi.org/10.4000/mots.1328 


\section{Les Ordonnances royales relatives aux juifs en Espagne et en France}

Poser la question des ordonnances prises par les rois d'Espagne et de France concernant les juifs, c'est effectuer deux constatations : la première, originelle, est que la parole de la loi met en discours dès le Pentateuque l'existence historique des Hébreux dans la verticalité sacrée du monothéisme (les énoncés les plus répétés, « Dieu dit », « Le Seigneur dit », «L'Éternel dit » confèrent une performativité absolue à des décrets « divins ») ; la seconde, ultérieure, est qu'une parole politique sur les juifs devenue légiférante, et à son tour religieuse, règle leur existence comme entité sociale singulière au sein de nations souveraines. Le Livre d'Esther illustre d'avance ce modèle :

Il est une nation répandue, disséminée parmi les autres nations dans toutes les provinces de ton Royaume; ces gens ont des lois qui diffèrent de celles de toute autre nation; quant aux lois du roi, ils ne les observent point, il n'est donc pas de l'intérêt du roi de les conserver. Si tel est le bon plaisir du roi, qu'il soit rendu un ordre écrit de les faire périr... (Livre d'Esther, versets 3, 8, 9, Bible traduite de l'hébreu par le Rabbinat français)

C'est donc la parole de la loi qui fonde l'existence historique et identitaire des juifs. Avec le passage de l'Empire païen à l'Empire chrétien de Constantin, une parole d’Église s'insère en tant qu'idéologie religieuse dans la souveraineté territoriale. Par la suite, avec la chute de Rome, cette parole maintenue renforce, dans la dimension doctrinale et administrative, les nouveaux royaumes créés, légitimant et unifiant en chacun le pouvoir royal. Telle est la fonction de la formule per gratia Dei rex (roi par la grâce de Dieu) qui accompagne la signature des ordonnances sur les juifs ${ }^{1}$.

1. L'analyse portera sur les textes suivants: pour l'Espagne, 1822, Lex Visigothorum, édition en langue romane du $13^{\mathrm{e}}$ siècle appelée Fuero Jurgo, Madrid, Real Academia de la Historia ; 1807 , Las Siete Partidas $\left(13^{\mathrm{e}} \mathrm{s}\right.$.), Madrid, Real Academia de la Historia; 1963, Concilios Visigóticos e Hispano-romanos, Madrid, José Vivés ; «Decreto de la expulsión de los judíos » de 1492, dans A. García-Gallo, 1959, Antología del derecho español, Madrid. Pour la France: M. de Laurière éd., 1723, Les Ordonances des Roys de France de la troisième Race, t. 1 à 6, Paris, Imprimerie royale; B. Leroy, 1998, Les Édits d'expulsion des juifs, Paris, Atlantica ; P. de Beaumanoir, 1970 [1283], Coutumes de Beauvaisis (13 ${ }^{\mathrm{e}} \mathrm{s}$.), reprint de l'édition de 1899-1900, Paris, Picard, 2 vol. ; "Décret d'émancipation », cité dans M. Winock, 2004, La France et les juifs de 1789 à nos jours, Paris, Le Seuil, p. 22 ; collectif, 1952, Les Constitutions et les principales lois politiques de la France depuis 1789, Paris, Librairie générale de droit et de jurisprudence.

Université d’Oviedo (Espagne), Faculté d'histoire 
À travers les différences que les siècles ont imposées à la production de ces lois et les diversités dues aux natures des entités politiques (royaumes féodaux, monarchiques, États nationaux), nous nous proposons de mettre en évidence aussi bien les traits communs constants et les imbrications discursives dans la récurrence du discours officiel tout au long de l'histoire des ordonnances, que les différences fondamentales entre deux traditions juridiques comparées, l'espagnole et la française, étant entendu que l'un des attributs de la souveraineté est d'édicter des lois qui catégorisent et qualifient des entités sociales fortement labellisées et marquées ${ }^{2}$, afin de justifier répressions imposées comme permissions octroyées.

\section{La parole de la loi catégorise le juif}

En droit, dans l'acte de langage édictant une loi, « les mots font tout ou presque [...], ils créent des choses et des faits (juridiques bien sûr, non pas matériels) ou les font disparaître sans trace ${ }^{3}$. Sur la base de ce principe, l'analyse conduit à constater qu'en Espagne ce sont les lois visigothes, à partir de la conversion du roi Recarede au catholicisme au milieu du $7^{\mathrm{e}}$ siècle, qui ont commencé à élaborer un droit commun, le Liber Judiciorum, pour tous les habitants du Royaume, avec la volonté d'imposer un ordre stable entre les Goths ariens (disciples d'Arius), les Goths catholiques et les Romains catholiques. Dans ce Liber, la parole politique sur les juifs (qui jusque-là suivait le codex de Rome) prend une place à part, autonome, dans le livre 12 «De devedar los tuertos e derraygar las sectas e sus dichos » [Pour déjouer les tromperies et déraciner les sectes et leur discours], et dans le titre 2 de ce livre intitulé «De los herejes e de los judíos e de las sectas» [Au sujet des hérétiques, des juifs et des sectes]. Cet ensemble de textes forme un corpus de dix-huit lois. L'analyse montre que les juifs en constituent le centre, étant directement concernés par seize d'entre elles. Le mot judíos se constate en effet dans les titres de ces seize lois. Dans les autres (la première et la deuxième), les juifs apparaissent en tant qu'individus mais à la fois masqués et marqués religieusement sous les noms de non fieles (infidèles) dans la première loi et de errados (égarés) dans la seconde. On trouve judíos en tant que seule catégorie visée par le contenu légal dans le texte de la première loi. Dans la seconde, bien qu'il ne soit pas prononcé, le nom s'inscrit en filigrane dans diverses accusations: déprécier les sacrements, mépriser l'Évangile, disputer contre la foi catholique, etc. Le juif est bien le véritable objet des lois sur les non fieles et les errados.

2. Sur les notions de labellisation, marquage et thématisation, voir M. Tournier, 2002, Des sources du sens. Propos d'étymologie sociale 3, Lyon, ENS Éditions, p. 32-46.

3. C. Grzegorezyk, 1986, «L'impact de la théorie des actes de langage dans le monde juridique. Essai de bilan », Théorie des actes de langage. Éthique et Droit, Paris, PUF, p. 186. 
Oubliant la tolérance d'Alphonse VII, qui se voulait au $12^{\mathrm{e}}$ siècle « prince des trois religions », Alphonse X «le Savant» (1252-1284), contemporain de SaintLouis, élabore le code dit des Siete Partidas, avec un double dessein : d'une part, unifier le Royaume et imposer un ordre nouveau sous sa souveraineté; d'autre part, effectuer une reconquête juridictionnelle du territoire hispanique. Cette volonté politique est engendrée par le «fait impérial» (el hecho del Imperio) qui découle de ce que le roi est élu, reçoit la couronne mais n'est pas reconnu comme tel dans les lois germaniques -, par les luttes de juridiction et par les questions dynastiques qui entourent le pouvoir. Dans ce nouveau code, la parole sur les juifs prend place au livre 7 (Partida 7, título 24, "Judíos ») et occupe 11 lois, les titres antérieurs (du $21^{\mathrm{e}}$ au $23^{\mathrm{e}}$ ) concernant les malédictions lancées contre les entremetteurs, ou ceux qui pèchent contre la nature, et les titres postérieurs (les $25^{\mathrm{e}}$ et $26^{\mathrm{e}}$ ) concernant les Maures et les hérétiques de confession chrétienne. Tous sont regroupés sous l'appellation religieuse de non fieles.

Au $15^{\mathrm{e}}$ siècle, la parole légale sur les juifs prend naissance dans les États généraux (Las Cortes del reino), sous la forme des lois de Valladolid (1411), puis des Cortes de Madrigal $(1476,1478)$ et de Tolède $(1480)$ qui renouvellent les lois de 1411 au sujet de l'imposition des quartiers fermés (juderias), de l'obligation de porter des signes d'infamie (chapeau très haut ou signaux cousus sur les vêtements), de l'interdiction d'exercer des fonctions publiques et d'utiliser des vêtements de luxe. Avec les Rois catholiques et la Reconquête menée à son terme au nom de Dieu, le thème de l'ordre nouveau devenu totalitaire, car territorial, politique, religieux, économique et social, se répand dans toute l'Espagne et jusqu'aux Amériques ${ }^{4}$. La parole légiférante sur les juifs s'exprime alors dans le décret qui ordonne leur expulsion, en 1492.

En corrélation avec ces conditions de production des textes, les désignations et les thèmes juridiques mis en œuvre à l'égard des juifs montrent que la parole de la loi agit toujours en référence à la foi catholique, laquelle fonde la souveraineté du roi-législateur, la légitime et la garantit. Tel est le rôle du performatif renouvelé d'édit en édit per gratia Dei et de la désignation récurrente non fieles. En Espagne, la définition du juif comme non-vrai sujet du roi découle de son « infidélité ». Cette logique juridique opère sans faille du $6^{\mathrm{e}}$ au $16^{\mathrm{e}}$ siècle. La voici à l'œuvre dans les Siete Partidas au $13^{\mathrm{e}}$ siècle : le juif y est défini comme «celui qui croit et obéit à la loi de Moïse, en suivant sa lettre même, et qui se circoncit et accomplit toutes les autres choses que sa loi lui commande $" 5$. Loi de Moïse et loi du Royaume sont considérées comme incompatibles. L'unité du Royaume en serait-elle menacée ? S'applique alors la valeur coercitive de la loi royale, en vertu du principe que «la coercitivité de la loi est le garant de l'unité

4. Les juifs sont les seuls habitants de l'Espagne auxquels les Rois catholiques interdisent d'aller aux Amériques.

5. "Judío es dicho aquel que cree et tiene la ley de Moysen segunt que suena la letra della et que se circunda et face les otres cosas que manda esa su ley », Loi 1 des Siete Partidas. 
et de l'unicité du pouvoir politique $»^{6}$. D'autant plus que «la loi humaine» au Moyen Âge agit «dans son lien avec la loi divine et la loi naturelle ${ }^{7}$.

Une raison vient corroborer cette logique juridique : la mémoire dite historique. Les juifs sont devenus ceux qui ont crucifié le Christ. Non-sujets et coupables, ils sont destinés à vivre en Espagne «comme en prison » (como en cautéverio), voire en servitude (servidumbre) : tel est leur « lignage» (linaje) ${ }^{8}$. Ainsi le texte même de la loi crée un « lignage juif », autrement dit une race. Absolument tous sont concernés, par effet de globalisation : d'où les nombreuses expressions, surtout dans les premières lois, de : los judíos, todos los judíos... Les interdictions se manifestent dans un sens totalitaire par l'emploi surabondant de la particule négative dans les titres des lois et par celui de l'indéfini négatif (ningun judío [aucun juif] atteint dix occurrences dans la Lex Visigothorum et dix dans les Siete Partidas). L’image du juif déicide par nature et filiation passe par là.

En outre, l'Édit d'expulsion de 1492, poursuivant cette logique du non-sujet coupable, utilise la désignation totalisante «judíos naturales o no naturales », qui établit une incompatibilité entre territoire du Royaume et existence des juifs. La notion de naturalité thématise le «problème» juif au Moyen Âge comme, plus tard, la « question » juive : la présence de juifs, supposés venus d'ailleurs, au sein d'une territorialité souveraine pose problème à la loi, laquelle, appliquée dans son intégralité, fait que leur existence doit être soumise soit à la conversion (thème des conversos dès le $7^{\mathrm{e}}$ siècle $\mathrm{e}^{9}$ ), soit à l'interdiction légale d'une identité juive, à l'intérieur comme à l'extérieur, donc à l'interdiction de la circoncision, de la Pâque juive, etc. ${ }^{10}$, soit au règlement de leur existence dans une sorte de captivité et d'isolement, «aussi longtemps qu'ils vivent parmi les chrétiens $»^{11}$, pour aboutir à leur pure et simple expulsion (Decreto de 1492). Au fur et à mesure que se renforce la loi du roi, la condition des juifs en Espagne empire, mais la logique qui conduira à cette expulsion est présente et active dès les conciles de Tolède et les ordonnances latines des rois visigoths, qui engendrent sur ce point une parole commune à l’Église et au souverain: "À la demande du concile, notre très glorieux seigneur [le roi Recarede] a en droit canon ordonné d'introduire... $»^{12}$. Les désignations judaeis, iudaeis babtizatis,

6. J. Quillet, 1988, « Note sur le concept médiéval de loi », Cahiers de philosophie politique et juridique, $\mathrm{n}^{\circ} 12$, La loi civile, Caen, Université de Caen, p. 54 .

7. Ibid., p. 45.

8. «Ellos vienen del linaje de aquellos que crucificaron a nuestro señor Jesucristo » [Ils descendent du lignage de ceux qui crucifièrent notre Seigneur Jésus-Christ], Loi 1 des Siete Partidas.

9. Qui exigerait d'autres développements impossibles à traiter dans le cadre de cet article.

10. "Que los judíos no se circunciden »; «Que los judíos non fagan su pascua segund su ley », Lex Visigothorum.

11. «En que manera deben facer su vida los judíos mientra viviesen entre los cristianos » (Las Siete Partidas).

12. "Suggerente concilio id gloriosissimus domnus noster canonibus inserendum praecepit», Canon 14, De iudaeis du $3^{\mathrm{e}}$ concile de Tolède, Consilios visigóticos, 1963, Madrid, José Vivés, p. 133 . 
infidelibus iudaeis, iudaeis conversis, inflexibilis iudaeorum perfidia (quatre occurrences), iudaeorum damnationem, plebs iudaeorum font écho aux thèmes conciliaires qui sont autant de refus d'admettre les juifs, même convertis et revenus de leurs "prévarications», en témoignage devant les tribunaux, en mariage mixte, aux offices publics, à la bénédiction des mets et à des repas pris en commun ${ }^{13}$, etc. L'exclusion du corps national (théologico-politique) a commencé par cette mise à l'écart systématique.

En France, les choses se présentent de manière très différente. La parole légiférante à l'égard des juifs s'effectue dans une longue suite récurrente d'« ordonnances ». Les conditions historiques de cette production d'édits royaux sont en rapport avec la féodalisation du Royaume, sa monarchisation puis sa déchéance. Elles apparaissent en particulier liées à la volonté des rois d'augmenter le trésor royal selon leurs besoins (conquêtes, croisades, fastes, etc.). Les désignations et les thèmes juridiques illustrent cette tendance.

Le discours de la loi va d'abord dans le sens juridictionnel de la féodalité : il fait du juif une sorte de serf au service de son seigneur. Là aussi, le «lignage » l'imposait. D'où la mobilisation par simple logique discursive de désignations telles que : nos juifs, son juif, son propre serf, les juifs du Roy, les juifs des Barons, le juif de notre domaine, les juifs de son Royaume. Cela pose le problème des "juifs» en termes de propriété et de finance; ils sont acceptés comme serfs ou locataires en vertu de services à rendre et de tributs à payer, soit au roi, soit aux seigneurs; ils « appartiennent » à un domaine. Chaque tribut à payer, de plus en plus exigeant et régulé, suppose l'octroi par le roi de lettres, privilèges et franchises qui leur permettent de vivre sur le territoire du Royaume.

On passera de la condition de serf à celle de juif patenté ou non patenté, donc à leur dépendance vis-à-vis de l'obtention de « Lettres patentes» et, bien sûr, du paiement des "redevances » qui en découlent. S'établit ainsi le statut d'une sorte de juif-marchandise qu'on trouve dans des ordonnances aussi diverses que les «Lettres patentes pour la Comtesse de Dreux», l'«Établissement des juifs à Melun», les "Privilèges et franchises accordées aux juifs demeurant dans le Royaume», la "Confirmation des Lettres de Charles V qui prolongeaient le temps pendant lequel il était permis aux juifs de demeurer dans le Royaume ", etc. La loi établit un lien explicite entre l'octroi du privilège pour les juifs d'habiter le Royaume, ou un domaine, et le rachat permanent du privilège. Ils ne sont pas des sujets du roi comme les autres. Ils peuvent même, en positif, jouir d'une protection spéciale, pourvu qu'ils la payent :

parquoy ils puissent vivre doresenavant soulz Nous en paix selon la teneur de leurs diz Privileges, et sans estre ainsi durement traictez, et souffrir les grands povretés et

13. De iudaeis conversis et post praevaricantibus ad testimonium non admitantur; De christianorum iudaeorum coniugiis; Ne iudaei officia publica agant; De frugibus fidelium ne a iudaeis benedicantur; De christianis qui cum iudaeis vescuntur, etc. 
miseres qu'ilz ont longuement souffert et par maintefois. Nous veillons prendre d'iceulx telle somme d'argent comme bon Nous semblera, et qu'ilz pourront bonnement supporter et paier. (Charles VI) ${ }^{14}$

Dans ce domaine, le prêt à intérêt leur est même permis par l'Église, la parole conciliaire ne l'interdisant qu'aux prêteurs et usuriers chrétiens. De là vient l'apparition de désignations comme: judaeis usuris, quae debentur judaeis (les choses qui sont dues aux juifs), debitis judeorum, debita judeorum, creditore judaeo, negotiis Judaerorum, etc., préfigurant l'image du juif prêteur et usurier, mots qui apparaissent souvent en cooccurrence ${ }^{15}$. Toutes les ordonnances, à partir de Philippe-Auguste, sont conçues dans cet esprit: elles tendent à protéger l'enrichissement des juifs pour donner au roi les moyens de les dépouiller ensuite. Voilà pourquoi, dès Philippe-Auguste, elles soumettent les juifs à l'officialisation ${ }^{16}$, au blocage puis à la confiscation de leurs créances. Les «Lettres touchant les Juifs » sont suivies par une ordonnance sur la confiscation des créances et l'ordre «que tous les Juifs vivent des labeurs de leurs mains » après expulsion ou sortie du Royaume ou domaine pour, ensuite, être rappelés à rejouer le rôle de prêteur-accumulateur. Il s'agit d'un modèle en boucle, qui se met en place avec Philippe-Auguste et se répètera, même sous Charles VI. Pragmatisme, voire cynisme, de la parole juridique au service du politique et de l'économique.

Il n'en demeure pas moins que les critères justificatifs, idéologiques, restent d'ordre religieux. Ils s'insèrent dans les titres des ordonnances et au sein des articles, ne serait-ce que sous les formes les plus symboliques, ainsi de l'obligation de porter les signes d'infamie (la rouelle) à laquelle l'«Ordenance» de Saint-Louis de 1269 donne force de loi :

C'est-à-savoir une roue de feutre ou de drap d'écarlate ${ }^{17}$, cousue sur la partie supérieure du vêtement, sur la poitrine et dans le dos, qui les fasse connaitre, que, de la roue, la largeur soit en circonférence de quatre doigts et que la concavité contienne une paume. (Louis IX) ${ }^{18}$

14. «Lettres qui accordent aux Juifs demeurans dans la Languedoil, absolution de tous les delits pour lesquels ils etoient poursuivis en Justice, et qui portent que dans la suite ils n'auront plus de Conservateur et qu'ils seront soûmis à la Jurisdiction du Prévost de Paris, des Baillis et Sénéchaux, et des autres Juges ordinaires », Les Ordonnances des rois de France de la troisième race, op. cit., t. 7, p. 643 .

15. Jusque dans des titres: «Ordonance qui porte que celle de Saint Louis contre les Usuriers et les juifs sera observée » (Philippe IV, 1299).

16. "Lettres qui portent que les juifs qui demeurent dans le Royaume seront obligez de prendre un grand Sceau, des Lettres de comission pour se faire payer de leurs dettes et des Lettres de permission de plaider par Procureurs " (Charles IV, 1388).

17. Nom d'une étoffe qui n'était pas forcément rouge. Le dictionnaire de Bescherelle (1845) relève des écarlates bleue, noire, verte. On remarque l'absence, dans la loi même, de la couleur jaune des condamnés par l'Inquisition. Le Dictionnaire de la conversation et de la lecture (W. Duckett éd., 1865) signale cependant qu'on peut préférer l'écarlate « avec une nuance jaunâtre ». Le mot, d'origine inconnue, indique un tissu chez Chrétien de Troyes : « cinq somiers... chargiez de robes et de dras, de boqueranz et d'escarlates », « robes de veir et d'erminetes... d'escarlate, grise ou de soie » (Érec et Énide, vers 1807 et 2061).

18. Les Ordonnances des rois de France, t. 1, p. 294. 
Ces prescriptions se renouvelleront d'édit en édit, tels des marqueurs textuels. C'est ainsi que Philippe III signe en 1283 un «Mandement par lequel le Roy ordonne que l'«Ordonance» de Saint Louis de l'an 1269 touchant les juifs sera exécutée » et que, dans ses "Lettres au sujet des juifs », Jean $1^{\text {er }}$ écrit en 1362: «lls seront obligez de porter une marque grande et placée dans un lieu où on puisse la voir afin qu'on puisse les distinguer des Chretiens ${ }^{19}$. De même les ordonnances interdisent-elles aux juifs de prendre en gage «aucun ornement d'Église, aucune vestement ensanglanté ou mouillé $»^{20}$. Plus centrale, en 1315, l'interdiction faite aux juifs de "disputer de la foy ${ }^{21}$, qui va se répétant par la suite, coupant les juifs de la vie intellectuelle : ils n'appartiennent donc pas au corps du Royaume et ne jouissent pas de la condition de sujets. Tout cela s'est répandu au-delà des édits royaux; le droit coutumier du $13^{\mathrm{e}}$ siècle en est témoin. Philippe de Beaumanoir a ainsi recensé dans ses Coutumes de Beauvaisis:

Cil ne doivent pas estre mis en tesmoignage qui sont hors de la foi crestienne, si comme cis qui est juis. Et aussi ne doivent pas estre receu en tesmoignage cil qui sont escommunié et renforcié [en état de péché mortel] (art. 1206) ; Doit chascuns savoir que nus ne doit espouser cele qui li apartient de lignage, [...] ne juise si ele n'est avant crestienne. (art. 585)

En somme, l' « infidélité » exclut la vérité, jusque dans les preuves de justice, de même qu'elle interdit tout mariage avec une juive, sauf si la conversion l'a précédé, etc. En bref, c'est la foi affichée qui donne la clé de la socialité.

Avec la Révolution française et la promulgation des Droits de l'Homme par la Constituante se dessine un nouvel ordre politique, qui suppose la déchéance à terme de l'Ancien Régime. Les décrets votés par cette Assemblée sont donc signés en 1790-1791 par Louis XVI en tant que chef d'État : il ne s'agit plus d'ordonnances royales proprement dites mais, par exemple, de «Lettres Patentes du Roi sur un Décret de l'Assemblée Nationale portant que les juifs connus en France sur le nom de Juifs Portugois ${ }^{22}$, Espagnols et Avignonnois, y jouiront des droits de Citoyen Actif», signées par «Louis par la grace de Dieu, et par la loi constitutionnelle de l'État, Roi des François » et débutant ainsi : «L'Assemblée Nationale [...] a decreté le 28 janvier, present mois, et Nous voulons et ordonnons ce qui suit... » (Registrées par la Cour des Aides, le 19 février 1790). Deux

19. Ibid., t. 1, p. 312 ; t. 3, p. 603. De même encore dans l'ordonnance de Louis $\mathrm{X}$ «pour le rappel des Juifs ", de 1315 (t. 1, p. 595, art. 14).

20. Ordenance du roi Philippe Auguste touchant les juifs (1218), t. 1, p. 35, art. 3.

21. Ordenance de Louis X (t. 1, art. 17).

22. En fait, juifs établis en Guyenne ou dans le comtat venaissin. Depuis Henri II, les juifs portugais sont officiellement appelés "les nouveaux chrétiens », la loi portugaise pratiquant la conversion obligatoire. Henri II les considère globalement comme convertis dans ses Lettres « par lesquelles donnons en mandement à nos amez et feaulx [...] ils fassent, souffrent et laissent lesdits Portugais dictzs Nouveaulx Chretiens jouyr et user plainement et paisiblement » (B. Leroy, Les Édits d'expulsion, op. cit., p. 91). 
pouvoirs légitimés parlent d'une seule voix: à la "grace de Dieu» est venu s'ajouter l'édiction légiférante de la Nation.

Dans le «Decret d'émancipation» voté le 27 septembre 1791, deux ans après le «droit de cité » reconnu aux protestants, la condition de citoyen actif n'est attribuée que par la révocation des actes juridiques antérieurs concernant les juifs. Ceux-ci vont donc être concernés, grâce aux articles 2 à 4 du titre 2 de la Constitution du 3 septembre 1791, par la priorité accordée au droit du sol : sont déclarés Français «ceux qui sont nés en France » ${ }^{23}$. Le label « juif » sous la dénomination d' «individus juifs » demeure dans le décret d'émancipation, mais à titre de constat et pour être évacué ; à l'établissement d'un statut particulier a succédé sa négation : le juif français sera le juif assimilé. Mais la révocation des ordonnances (et de la « redevance levée sur les juifs ») a pour contrepartie que ce serment «sera regardé comme une renonciation à tous privilèges et exceptions introduits précédemment en leur faveur »... y compris la renonciation aux intérêts des prêts consentis ${ }^{24}$.

Toute la géométrie juridique se trouve ainsi renversée : à la verticalité de la catégorisation, en bas d'échelle, s'oppose l'horizontalité de l'égalité juridique des citoyens majeurs. Les marquages de fidélité, eux, se déplacent vers l'acte performatif de tout citoyen, la sacralité se retrouvant dans le serment prêté devant trois entités mises sur le même plan:

Je jure d'être fidèle à la Nation, à la Loi et au Roi [je souligne] et de maintenir de tout mon pouvoir la Constitution du Royaume, décretée par l'Assemblée Nationale constituante aux années 1789, 1790 et 1791. (art. 5 de la Constitution de 1791)

Ainsi décatégorisé juridiquement, le juif n'a plus de thèmes ni de qualifications qui lui soient propres.

\section{La parole de la loi qualifie le juif}

Dans l'acte juridique de l'édiction d'une loi, la première proposition sert à établir la raison d'être de cette loi. Cela se matérialise dans le texte par des thématisations et des marquages qui justifient la création d'un nouvel état de choses légal. Cette proposition remplit la fonction d'attribuer à un sujet, à un objet, à une situation ou à une action certaines qualités et qualifications juridiques ${ }^{25}$. Sur cette base, l'analyse des lois sur les juifs de la Lex Visogothorum permet de constater que la raison avancée pour la constitution de cette législation est

23. Les Constitutions et les principales lois politiques de la France, op. cit., p. 5 .

24. Le jacobin antisémite Reubell ne manque pas de rappeler à l'Assemblée l'« oppression usurière » exercée sur le peuple par les juifs d'Alsace et de Metz.

25. Voir C. Grzegorczyk, op. cit., p. 189. Voir aussi P. Jestaz, 1998, «La qualification en droit civil», Droits, $\mathrm{n}^{\circ}$ 18, La qualification, Paris, PUF, p. 52. 
contenue dans le texte des quatre premières lois. On y lit, comme proposition initiale, que le devoir des rois est de se protéger des «erreurs» des juifs, de les forcer à corriger leur désir de «faire du mal» et de maintenir dans l'ordre le "peuple fidèle», afin qu'à partir de là celui-ci comprenne que les lois sont bonnes. La seconde proposition affirme que les rois doivent légiférer contre leurs ennemis, les «infidèles », afin d'instaurer la paix dans le peuple de Dieu en stigmatisant les «fautes ${ }^{26}$ des juifs. Une troisième proposition dit que les lois sur les juifs sont engendrées par ce qu'il y a de mauvais dans les juifs euxmêmes, à cause de leur perfidie, leur volonté de tromper, leur pouvoir et leur influence. Outre leurs erreurs religieuses (yerros), la loi attribue aux juifs certains traits moraux: perfidia, maldade (iniquité et méchanceté), enganno (malhonnêteté), etc. Tout se passe comme si, en refusant l'obligation décrétée par le roi Recarede de se faire baptiser, les juifs avaient signé, par leurs «vices», leur exclusion juridique du Royaume. Les lois sur les juifs découlent de ces propositions : ils ont rendu le Royaume «sale» (sucio) ${ }^{27}$. À partir de telles affirmations, l'argumentation des ordonnances s'efforce d'établir une «infidélité » aussi salissante en tant qu'état légal, c'est-à-dire objet nécessaire d'actes juridiques, et de la punir pour ce qu'elle entraine de mauvais ${ }^{28}$, de définir, donc, les critères qui permettent de légiférer à son propos. Omniprésence de la stigmatisation au sein même de la loi: les non fieles, herejes et autres errados submergent les expressions disant la foi (los fieles, la fé de los fieles, la fé de Dios, los fieles de Dios, los fieles de Cristo) et le nom de judios (65 occurrences) l'emporte en fréquence sur cristianos (58 occurrences), telle une incantation de phrase en phrase plus prégnante. Les labels agissent comme des marqueurs.

Dans Las Siete Partidas, il est dit en outre que la raison originelle pour laquelle Église, rois et princes ont permis aux juifs d'habiter le Royaume est qu'ils sont les porteurs d'une mémoire, puisqu'ils sont de la race (linaje) de ceux qui ont crucifié le Christ. Affirmation dans la négation: les qualifications établissent leur état légal en tant que peuple maudit, peuple élu (« privillegiados») mais perdu d'honneur ("perdiesen la honra ») 29 . En vertu de ce principe retors, on se doit de reconnaitre leur existence, mais dans un isolement à organiser, des juderias aux signalements, aux interdits, à la prophylaxie qui doivent les séparer des chrétiens : l'infidélité est une lèpre.

Le décret d'expulsion des Reyes catolicos (1492) affirmera que ces mesures d'isolement n'ont pas été suivies de succès pour ce qui est de la coupure de

26. La loi qualifie, par exemple, de « fautes » la Pâque juive, le mariage juif, etc.

27. «Por la maldade de los judíos solamente entendemos que el nuestro regno es ensuciado » [Par iniquité des juifs nous disons seulement que notre règne est sali par eux], Lex Visigothorum, loi 3.

28. "Por ende, es derecho, que la sucidumbre [le vice d'infidélité] que es mas sucia que todas las otras sucidumbres é demas yerro, deve seer desfècha y echada [doit être effacé et rejeté] de entre los cristianos ", Lex Visigothorum, loi 8.

29. Las Siete Partidas, loi 3. 
toute communication entre juifs et chrétiens et qu'il faut en finir avec la parole juive, qui risque de pervertir le peuple: «[Les juifs] recherchent toujours tous les moyens de pervertir et d'éloigner de notre sainte foi catholique les fidèles chrétiens, de les en écarter et de les entrainer dans leur croyance damnable. » Se dessine alors un grand mouvement "pour la paix» et "la sainte foi catholique », qui considère les juifs comme « indésirables et périlleux pour la république » : l'expulsion serait imposée par la parole même des juifs. Les attributs des juifs sont alors exprimés par les syntagmes suivants : comunicación de los judíos (2 occurrences), aljama (assemblée) del judío (2 occurrences), judíos (7 occurrences), dichos y sentencias de los judios, conversacion del judío...

Des traits communs de récurrence discursive tracent et chargent le portrait du juif dans les édits royaux : à la « paix du Royaume » et à la «pureté de la foi », qui demeurent, s'ajoute l'affirmation de la primauté d'une protection nationale des Espagnols; aussi les marqueurs qualificatifs découlant de la notion de « race juive » s'amplifient-ils. Ce sont d'ailleurs toujours les mêmes : la perfidie, la saleté, la manipulation, la mauvaiseté, la richesse, la tromperie, que vient renforcer le «pouvoir de perversion », qualifications qui fonctionnent en opposition aux bonnes entités: le roi, le Royaume, la foi, le canon, la nationalité espagnole, qui fondent, unifient et justifient les mots d'ordre. Toutes situent les juifs en dehors du corps social, politique, économique, juridique et culturel de la nation et du territoire.

Cette mise à part va plus loin : le juif est à chasser d'Espagne. Dans le décret d'expulsion totale des juifs de 1492, les commandements deviennent encore plus radicaux : la punition de la transgression est la mort avec confiscation des biens. S'ajoute l'interdiction faite à tous les Espagnols de donner protection aux juifs, sous peine d'emprisonnement et de confiscation des biens. Les garanties faites aux juifs se bornent à la possibilité de vendre leurs biens et de quitter le Royaume, mais "sans or, argent ni monnaie», ni aucune richesse. L'emploi du performatif mandar (commander) signe en Espagne l'absolu de la parole légale ${ }^{30}$.

On ne sera pas surpris, vu la catégorisation économique des juifs dans les ordonnances françaises qui régissent leur "establissement», de voir que le réseau qualificatif y est, sauf exception, moindre qu'en Espagne. Certes, on y retrouve certaines des argumentations espagnoles (combat contre le mal, paix du Royaume, «reformation des mœurs »), mais le juif y est qualifié, pour l'essentiel, vu sa fonction de prêteur, par ses capacités d'enrichissement. Au texte

30. Il en est ainsi au Portugal, à la même époque, où le décret d'expulsion de 1496 de ces filhos de maldiçam (fils de malédiction) insiste sur le fait que «les juifs sont obstinément opposés à notre sainte foi catholique envers le Christ Notre Seigneur» et «qu'ils ont commis et commettent toujours contre cette foi de grands maux et blasphèmes" (Lettre Patente por que os Judeus de Portugal se sayan destes regnos e nom morem nem esteen nelles [... que les juifs du Portugal s'en aillent du Royaume et n'y demeurent plus jamais]). Édit peu appliqué. 
des ordonnances de Louis IX condamnant les usuriers et à celles qui en renouvellent l'application, l'ordonnance de Philippe IV Le Bel de 1299 mêle « la promotion des vertus, l'extermination des vices, la tranquillité de nos sujets et l'établissement de la paix ${ }^{31}$; mais l'accusation d'infidélité et de déicide ainsi que les qualifications morales et religieuses péjoratives, qui abondent dans les lois espagnoles, sont relativement rares. On découvre d'ailleurs, dans le titre d'une "Ordenance» de 1302, une protection du roi sur les juifs vis-à-vis des Inquisiteurs de la foi32. Quelques décrets, cependant, font état de motivations de type religieux plus affirmées, tel celui de Louis XII pour la Provence : la fréquentation des juifs y est vue comme néfaste. Formant État dans l'État, «ladite secte et nation judaïque » ne fait qu'engendrer hérésies et mauvais exemples:

Pour l'honneur et reverance de Dieu notre créateur et exaltation et conservation de notre foy et religion chretienne nous qui sommes roy très chrétien et à qui notre createur a fait de sy grans graces nectoyer tous nos dits pays de telle secte et nation pour obvier auxdites inconvenients qui, comme dit est, par ladite frecantation et communication en pourroient advenir. (Lettre de Louis XII ordonnant l'expulsion des juifs de Provence, 1500)

Comme on le voit avec cet exemple, c'est au moment des expulsions (souvent momentanées ${ }^{33}$ ) que les justifications avancées retrouvent des arguments religieux et quelques marqueurs traditionnels (notre foy) mêlés à des accusations plus laïques (crimes, délits, délinquance, désobéissance). Les «Lettres»de Charles VI donnent ainsi pour raisons légales à une « sortie du Royaume » :

des exces et desliz que les diz juifs faisoient et font chacun jour sur les Chretiens [...] d'avoir commis et perpetré plusieurs crimes excés et deliz et en maintes manieres avoir délinqué, especialement contre notre foy, et aussi contre le contenu en noz dictes Lettres à eux octroyees (Lettres qui portent que celles par lesquelles il est ordonné que tous les juifs sortiront du Royaume seront exécutées..., 1388).

Mesurée à l'aune des siècles, la comparaison des ordonnances royales montre que c'est en Espagne surtout que la parole antijuive a catégorisé et qualifié l'existence des juifs puis leur non-existence à l'aide d'un appareil argumentatif de type essentiellement religieux, justifiant des réoccupations d'espace. La récurrence que l'on constate dans ces édits aboutit à la mise en place d'une interdiction absolue : suppression de quelque droit de territorialité que ce soit, de toute tolérance religieuse, de toute nationalité (sauf cas de conversion, le

31. "ad promotionem virtutum et exterminium vitiorum ac subditorum nostrorum tranquillitatem, et pacem statutam », Ordonnances des rois de France, t. 1, p. 333.

32. "Ordenance touchant les Inquisiteurs de la foi portant qu'ils ne pourront poursuivre les juifs pour usures, pour sortilèges ni pour tous autres crimes qui ne sont pas de leur compétence ».

33. "Ordonance portant que les juifs venus d'Angleterre et de Gascogne seront expulsez du Royaume à compter de ce Parlement jusqu'a Caresme suivant » (Philippe Le Bel, 1302). 
juif ne peut être, par définition, qu'apatride), interdits multiples, confiscation des biens, expropriation générale, coupure de tout lien social. En comparaison, les ordonnances des rois de France apparaissent plus pragmatiques, avec des expulsions locales ou courtes et des rappels intéressés ${ }^{34}$. Cela ne peut cependant faire oublier le substrat religieux qui préside en Europe à la condition particulière faite aux juifs par des rois chrétiens et « de droit divin ». C'est dans cette veine d'un « lignage juif» (qui trouvera d'autres définitions, plus physiquement racistes) que viendront s'inscrire les lois de Nuremberg et certains décrets de Vichy. Les traits communs à l'ensemble des textes constituent une véritable grammaire, celle d'un discours totalisant (les juifs pris en tant que «nation» étrangère au pays) et totalitaire (un roi, une loi, une foi) qui, dans certaines mentalités occidentales, a précédé les volontés étatiques d'un anéantissement total des juifs et autres communautés indésirables. Tant il est évident qu'audelà de la loi qui en est imprégnée, l'esprit de l'Inquisition aura préludé à la Shoah. 